\title{
GASTOS AMBULATORIAIS DO SUS EM SERVIÇOS AUDITIVOS NAS MACRORREGIONAIS DO ESTADO PARANÁ
}

\author{
Robsmeire Calvo Melo Zurita ${ }^{1}$, Rebeca Melo Zurita ${ }^{2}$ \\ ${ }^{1}$ Universidade Estadual de Maringá (UEM). Doutoranda em Enfermagem, Maringá - PR. ${ }^{2}$ Universidade Federal do \\ Mato Grosso (UFMT). Curso de Medicina, MT. E-mail: $\underline{\text { robszurita@bol.combr }}$
}

\begin{abstract}
RESUMO
A deficiência auditiva é considerada uma doença severa e incapacitante que acomete 3,3\% da população brasileira e pode levar a uma sobrecarga tanto social quanto econômica ao indivíduo portador, a sua família e a sociedade. Faz parte da Política Nacional de Atenção a Saúde Auditiva o atendimento integral aos usuários do Sistema Único de Saúde (SUS), que compreende desde ações que englobam a atenção básica (promoção, prevenção e identificação precoce de problemas auditivos) até as de alta complexidade como protetização, em serviços credenciados. 0 objetivo do estudo foi estimar os gastos de procedimentos realizados na alta complexidade da Saúde Auditiva pelas macrorregionais no Estado do Paraná em 2005 e 2006 nos serviços credenciados pelo Ministério da Saúde. Estudo descritivo, documental, quantitativo das informações secundárias coletadas do Sistema de Informação Ambulatorial do SUS (SIA/SUS) das instituições públicas, privadas e filantrópicas. Os gastos com a Saúde Auditiva no Estado do Paraná concentraram-se em $63 \%$ no setor privado seguido pelos serviços filantrópicos com $27 \%$ e os serviços públicos com $10 \%$ dos recursos financeiros.
\end{abstract}

Palavras chaves: Gastos em Saúde. Perda Auditiva. Custos de Cuidados de Saúde.

\section{SUS EXPENSES IN THE OUTPATIENT SERVICES HEARING IN PARANA STATE MACRO REGIONS}

\begin{abstract}
Hearing impairment is considered an severe and disabling disease that affects $3.3 \%$ of the Brazilian population and can lead to both, social and economic overburden to the hearing impaired person, their family and society. It is part of the National Policy for Hearing Health the integral care to users of the Health System (HS), which extends from actions that include primary health care (promotion, prevention and early identification of hearing problems) until to the high complexity as prosthesis, in accredited services. The aim of this study was to estimate the cost of procedures performed in the high complexity of Hearing Health by regions in the State of Paraná in 2005 and 2006 services accredited by the Ministry of Health. Descriptive and documental study, quantitative information collected from the secondary system Outpatient Information SUS (SIA / SUS) of public, private and philanthropic. Spending on Health Hearing on the State of Paraná focused on $63 \%$ from private philanthropy followed by $27 \%$ and utilities with $10 \%$ of the funds.

Keywords: Health Expenditures. Hearing Loss. Health Care Costs.
\end{abstract}




\section{INTRODUÇÃO}

A surdez e a deficiência auditiva são doenças incapacitantes que podem levar a uma sobrecarga tanto social quanto econômica ao indivíduo portador, bem como a sua família e a sociedade ${ }^{(1)}$.

Além da idade, a perda auditiva pode estar associada a diversos fatores, tais como traumatismo craniano, exposição extra ocupacional ao ruído, tabagismo, doenças sistêmicas, história familiar de déficit auditivo e exposição a agentes químicos ${ }^{(2)}$.

De acordo com a Organização Mundial da Saúde (OMS), são consideradas idosas as pessoas com mais de 65 anos. Este referencial, entretanto, é válido para habitantes de países desenvolvidos. Nos países em desenvolvimento, como o Brasil, a terceira idade começa aos 60 anos. A estimativa era de que no ano de 2000, houvessem aproximadamente 2,25 milhões de brasileiros com dificuldades auditivas e 3,3\% da população brasileira declararam ter algum problema auditivo, dificuldade ou incapacidade de ouvir ${ }^{(3)}$.

Com o objetivo de desenvolver ações nos três níveis de assistência à saúde o Ministério da Saúde (MS) instituiu a Política Nacional de Atenção a Saúde Auditiva no Sistema Único de Saúde (SUS) em 2000 com a normatização através de portarias que prevê o atendimento integral aos usuários, com ações que englobam a atenção básica (promoção, prevenção e identificação precoce de problemas auditivos), serviços credenciados de média complexidade e alta complexidade realizando a triagem, o diagnóstico, avaliação, seleção, tratamento clínico, protetização, acompanhamento, reabilitação e terapia fonoaudiológica ${ }^{(4)}$.

A Portaria $n^{\circ} 432$ SAS/MS de 14 de novembro de 2000 visando regulamentar, no âmbito ambulatorial, o diagnóstico, o acompanhamento e a protetização de pessoas com deficiência auditiva no nível da alta complexidade, excluiu os procedimentos de reabilitação auditiva do Sistema de Informação Hospitalar (SIH-SUS) incluindo-os na tabela de serviços e classificação do Sistema de Informação Ambulatorial (SIASUS), códigos e procedimentos na APAC-SIA (Autorização de Procedimento de Alta Complexidade- Sistema de Informações Ambulatoriais), procedimentos ambulatoriais e de alto custo. Foram incluídos 43 procedimentos novos de diagnóstico, de acompanhamento, de seleção e fornecimento de próteses auditivas, com financiamento do Fundo de Ações Estratégicas e Compensação (FAEC) ${ }^{(2)}$.

O cadastramento e a habilitação para o funcionamento das Unidades Prestadoras de Serviços (UPS) na área de saúde auditiva foi designado de forma integral, para o Estado do Paraná bem como a definição da rede de assistência. Conforme a Política 
Nacional de Atenção à Saúde Auditiva a Portaria GM/MS n² 2073 de 28 de setembro de 2004 considera a possibilidade de êxito de intervenção na história natural da deficiência auditiva por meio de ações de promoção e de prevenção em todos os níveis de atenção à saúde, por intermédio de equipe multiprofissional e interdisciplinar, utilizando-se de métodos e técnicas terapêuticas específicas ${ }^{(5)}$.

A portaria SAS/MS $\mathrm{n}^{\circ} 587$ de 7 de outubro de $2004^{(6)}$ preconizou 1 serviço a cada 1.500.000 habitantes, no Estado do Paraná e desta forma seriam implantados 07 Serviços de Saúde Auditiva. A Secretaria Estadual de Saúde do Paraná encaminhou a relação dos serviços aprovados após pactuação na Comissão de Intergestores Bipartite (CIB), onde houveram a implantação de 20 Serviços de Saúde Auditiva, bem como a redistribuição dos recursos físicos e financeiro para cada serviço que seria habilitado.

No Brasil, a compra de serviços de saúde, pelo setor público, acompanhou a grande expansão da oferta de serviços privados de assistência hospitalar ocorrida na década de 1970 financiada pelo Estado. A compra dos serviços era realizada de forma desordenada, conforme a oferta da iniciativa privada, não sendo, portanto, consideradas as necessidades da população, como também foi desordenada a expansão dos serviços, sem planejamento e avaliação. O governo federal, por meio do Instituto Nacional de Previdência Social (INAMPS), tornou-se o maior comprador de serviços de saúde, sem a participação das Secretarias Estaduais e Municipais de Saúde ${ }^{(7)}$.

A alta complexidade dos serviços auditivos tem que ser articulada com as diferentes portas de entrada dos usuários SUS aos serviços. A rede de atenção à saúde tem que promover as condições necessárias a cada caso, garantido, desta forma, a continuidade da assistência desde o primeiro atendimento até a resolução da demanda. A integração dos serviços de saúde refere-se à necessidade de se ofertar atendimentos e serviços para determinada população em determinado território e sob uma única gestão, a fim de garantir a continuidade do cuidado durante todo o ciclo de uma condição de saúde ou de uma doença ${ }^{(8)}$.

Este estudo tem como objetivo estimar a frequência e os gastos com procedimentos realizados na alta complexidade da Saúde Auditiva pelas macrorregionais no estado do Paraná em 2005 e 2006 nos serviços credenciados pelo Ministério da Saúde (MS).

\section{MATERIAIS E MÉTODOS}

Estudo descritivo, documental com abordagem quantitativa. O campo de pesquisa abrange as informações secundárias 
das instituições prestadoras de serviços credenciados pelo MS no Estado do Paraná no período de 2005 e 2006. No final do ano de 2007, ocorreu a unificação dos procedimentos ambulatoriais e hospitalares em uma tabela única de procedimentos do SUS - Sistema de Gerenciamento da Tabela de Procedimentos, Medicamentos e Órtese Prótese e Materiais Especiais (OPM) do SUS (SIGTAP) definindo os anos da pesquisa realizada. Os dados foram coletados do SIA/SUS, Banco de Dados do Departamento de Informação do SUS (DATASUS), e tabulados pelo Programa Tabwin versão 3.5 na Secretaria Municipal de Saúde do munícipio de Maringá.

Os dados foram coletados entre os meses de setembro a dezembro de 2007 e foi solicitada e autorizada a coleta de dados ao Centro de Formação e Capacitação Permanente dos Trabalhadores da Saúde da Secretaria Municipal da Saúde do município de Maringá (CECAPS) através do oficio $\mathrm{n}$ 은 2008/2008, da Gerência de Auditoria Controle e Avaliação (GACA). Após a coleta, os dados brutos foram distribuídos e organizados em planilhas do programa Excel 2003 para serem agrupados pelas macrorregionais.

O Estado do Paraná conta com 22 Regionais de Saúde (RS) que constituem a instância administrativa intermediária, estruturadas nas seis macrorregionais:
Metropolitana que agrega as $1 \underline{a}$ e $2 \underline{a}$ RS;

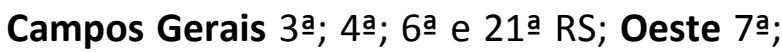

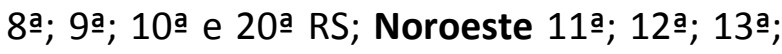
14a e 15a RS; Norte 16무 ; 17a; $18^{a}$ e 19a RS e a macrorregional Centro com a 5a e 22a RS.

$\mathrm{O}$ valor gasto per capita foi calculado dividindo-se o total gasto pela população referenciada da macrorregional onde a Unidade Prestadora de Serviço (UPS) estava habilitada. Para a definição das UPS foi utilizado o Cadastro Nacional de Estabelecimento de Saúde (CNES) cuja base operacional é de domínio público e propicia o conhecimento da realidade das condições de infra- estrutura e da rede assistencial ambulatorial existente por tipo de prestador, a natureza e a esfera administrativa.

O projeto foi submetido ao Comitê Permanente de Ética em Pesquisa Envolvendo Seres Humanos da Universidade Estadual de Maringá e aprovado com parecer no 556/2008 conforme previsto pela Resolução 466/2012. O TCLE não foi utilizado visto serem dados secundários.

\section{RESULTADOS E DISCUSSÃO}

No ano de 2005 o Estado do Paraná contava com 24 serviços credenciados ao SUS e a partir de 2006, com o estabelecimento da nova rede aprovada na Comissão Intergestores Bipartite (CIB) ocorreu uma redistribuição de cotas de produção e teto de financiamento, por prestador de acordo com 
os critérios de área de abrangência e complexidade (média e alta complexidade), tendo como referência a população (per capita). Em 2006, esse número foi reduzido para 20 serviços, seguindo a política nacional de redução financeira. No mesmo ano, o MS diminuiu o teto financeiro do FAEC passando ao teto limitado por produção da Média e
Alta Complexidade (MAC). No Brasil em 2006 foram habilitados pelo MS, 51 serviços sendo que 26 de alta complexidade e 25 de média complexidade $^{(9)}$. Os serviços foram descredenciados porque não conseguiram atender ao preconizado e normatizado pelas portarias vigentes não havendo a habilitação dos serviços (Tabela 1).

Tabela 1. Produção ambulatorial e gastos na saúde auditiva segundo prestadores do Estado do Paraná, 2005 e 2006.

\begin{tabular}{lcccccc}
\hline & \multicolumn{2}{c}{2005} & \multicolumn{2}{c}{2006} & \multicolumn{2}{c}{ Total Geral } \\
\cline { 2 - 7 } Tipo de Prestador & Freq. & Gasto R\$ & Freq. & Gasto R\$ & Freq. & Gasto R\$ \\
\hline Privado & 43716 & $16.174 .975,13$ & 33679 & $10.779 .887,13$ & 77395 & $26.954 .862,26$ \\
Filantrópico & 26798 & $7.276 .364,34$ & 21640 & $4.433 .612,57$ & 48438 & $11.709 .976,91$ \\
Público & 4554 & $2.019 .989,70$ & 5858 & $2.113 .891,75$ & 10412 & $4.133 .881,45$ \\
\hline Total & $\mathbf{7 5 0 6 8}$ & $\mathbf{2 5 . 4 7 1 . 3 2 9 , 1 7}$ & $\mathbf{6 1 1 7 7}$ & $\mathbf{1 7 . 3 2 7 . 3 9 1 , 4 5}$ & $\mathbf{1 3 6 2 4 5}$ & $\mathbf{4 2 . 7 9 8 . 7 2 0 , 6 2}$ \\
\hline
\end{tabular}

Fonte: SIA-SUS/CNES/2007.]

O art. 197 da Constituição Federal de 1988 declara que a saúde é um serviço de utilidade pública e, portanto, pode ser prestado pela iniciativa privada, mas sujeito às normas, ao controle e à fiscalização do poder público. No art. 199 reitera que a iniciativa privada participará do SUS como uma rede de serviço complementar segundo diretrizes deste, mediante contrato de direito público ou convênio, tendo preferência as entidades filantrópicas e as sem fins lucrativos ${ }^{(10)}$.

Analisando a Tabela 1, no ano de 2006, verificou-se que houve redução nos gastos e que cinco instituições não apresentaram produção nos bancos pesquisados neste referido ano. A análise dos gastos pelas UPS, observou que para as filantrópicas o ano de 2006 em relação ao ano de 2005, apresentou uma redução de R\$ 2.842.751,80. Para o prestador privado, houve redução no financeiro, esta diferença foi de $\mathrm{R} \$ 5.395 .088,00$ e para o prestador público um aumento de $\mathrm{R} \$ 93.902,05$.

$\mathrm{Na}$ análise dos gastos direcionados a saúde auditiva observou-se que o setor privado utilizou, aproximadamente, $63 \%$ dos recursos destinados a saúde auditiva, os serviços filantrópicos utilizaram em torno de $27 \%$ e os serviços públicos utilizaram aproximadamente $10 \%$ dos recursos financeiros (Tabela 1). 
A ausência de regulamentação da portaria no 432/2000 e o aumento dos valores dos procedimentos relativos às próteses auditivas acarretaram no aumento crescente da demanda ao MS, aos Estados e Municípios, de clínicas privadas e de representantes comerciais de empresas que comercializavam aparelhos auditivos buscando sua inserção no SUS ${ }^{(11)}$.

O valor dos aparelhos auditivos apresentou a primeira redução em 2002 fundamentada pela portaria $n=2035$ /GM de 4 de novembro de 2002 , e em face à variação cambial reajustou os valores dos procedimentos relativos às próteses auditivas da tabela SIA-SUS que variavam entre $\mathrm{R} \$$ 523,00 a $\mathrm{R} \$ 2.548,00^{(12)}$.

A portaria GM/MS n² 2073 em 28 de setembro de 2004 reduziu os valores dos procedimentos relativos à área, dentre eles as próteses auditivas que receberam nova classificação, anteriormente classificadas por modelo e tecnologia. Foram descritas e classificadas por A, B e C para os Aparelhos de Amplificação Sonora e Individual (AASI) que são equipamentos eletrônicos, para a percepção de fala, que necessitam de assistência técnica especializada que garantam a durabilidade e a manutenção da qualidade acústica(5).

A partir de 2005, passaram a vigorar os novos valores para os procedimentos relativos a saúde auditiva, e as próteses auditivas fixadas a três tipos com valores fixos de $R \$ 1.050,00(A), R \$ 1.400,00$ (B) e $R \$$ 2.200,00 (C). Em 2007 o valor sofreu variação de $50 \%$ (portaria no $308 /$ SAS de 10 de maio de 2007), pois os aparelhos tipo A apresentaram valor fixado em $\mathrm{R} \$ 525,00$; para o tipo B em $\mathrm{R} \$ \mathbf{7 0 0 , 0 0}$ e para o tipo C em $\operatorname{R} 1400,00$. A redução indica que houve um viés em prol de aparelhos mais caros sem justificativa para esse fato ${ }^{(11)}$.

Segundo os dados do MS em Nota técnica Informativa $n^{\circ}$ 004, no ano de 2003 no Brasil, os gastos com saúde auditiva foram de $\mathrm{R} \$$ 118.276.989,75, sendo que $R \$$ 103.371.561,75, ou seja, $87,39 \%$ foram destinados ao fornecimento das próteses auditivas. Em 2004 o custo foi de R\$ 183.401.837,04 com 588.464 procedimentos. Destes procedimentos $96.986(88,7 \%)$ foram com próteses auditivas ${ }^{(12)}$.

Em 2005, no Brasil, o gasto com a saúde auditiva ambulatorial, foi de $\mathrm{R} \$ 166.621 .722,99$ (839.725 procedimentos), sendo $\mathrm{R} \$$ 153.976.150,00 utilizados em 113.983 próteses auditivas. O Estado do Paraná gastou $\mathrm{R} \$ \mathbf{2 5}$ 2571.329,00, aproximadamente $15,30 \%$ do total do recurso financeiro com procedimentos auditivos. Em 2006, até a competência de outubro, o gasto com saúde auditiva ambulatorial foi de $\mathrm{R} \$ 131.854 .698,04$ (804.967 procedimentos) sendo que R\$ 120.114,35 (85.871 próteses auditivas) sendo 
$91,10 \%$ do valor total com protetização. Nesse mesmo ano, o número de acompanhamentos e terapias fonoaudiológicas corresponderam respectivamente a $5,75 \%$ e $14,11 \%$ do total de procedimentos realizados. De acordo com as estratégias da Política Nacional de Saúde Auditiva, o processo de reabilitação audiológica envolve trabalho amplo e multiprofissional que prevê a adaptação de próteses auditivas de fluxo contínuo, com acompanhamento médico, fonoaudiológico, psicológico, da assistência social e da enfermagem, tanto para ajustes como para verificações periódicas das condições técnicas, físicas e sociais do benefício obtido com o uso das próteses auditivas ${ }^{(12,13)}$.

A regionalização, como estratégia para a construção uma rede intermunicipal e interestadual, hierarquizada e de referência especializada, organiza os fluxos de referência e contra-referência, assim como a implantação de instrumentos e estratégias que permitem o acesso de usuários aos serviços de saúde, trazendo para a pauta o conceito de regulação assistencial, em substituição às proposições de implantação das centrais de controle de leitos, consultas e exames $^{(9)}$.

A macrorregional de menor valor per capita é a do Centro, justificada por um único serviço e por ser a área de menor concentração populacional do Estado do Paraná, inferindo que o acesso pode se tornar mais difícil para os usuários .

Tabela 2. Distribuição dos serviços auditivos por macrorregião no estado do Paraná, 2005 .

\begin{tabular}{|c|c|c|c|c|c|c|c|}
\hline Macrorregional & Freq & $\%$ & Gasto & $\%$ & População & $\%$ & $\begin{array}{c}\text { Gasto per } \\
\text { capita }\end{array}$ \\
\hline $\begin{array}{l}\text { Metropolitana } \\
\text { (5 serviços) }\end{array}$ & 26113 & 34,78 & $7.672 .773,41$ & 30,12 & 3508388 & 34,19 & 2,1869 \\
\hline $\begin{array}{l}\text { Campos Gerais } \\
\text { (3 serviços) }\end{array}$ & 7721 & 10,30 & $1.744 .355,33$ & 6,85 & 1048390 & 10,21 & 1,6638 \\
\hline $\begin{array}{l}\text { Oeste } \\
\text { (6 serviços) }\end{array}$ & 16826 & 22,41 & $5.249 .991,09$ & 20,61 & 1806276 & 17,60 & 2,9065 \\
\hline $\begin{array}{l}\text { Noroeste } \\
\text { (4 serviços) }\end{array}$ & 6992 & 9,31 & $3.618 .511,38$ & 14,20 & 1637963 & 15,97 & 2,2091 \\
\hline $\begin{array}{l}\text { Norte } \\
\text { (5 serviços) }\end{array}$ & 13472 & 17,95 & $5.697 .713,19$ & 22,37 & 1674543 & 16,32 & 3,4025 \\
\hline $\begin{array}{l}\text { Centro } \\
\text { (1 serviço) }\end{array}$ & 3944 & 5,25 & $1.487 .984,77$ & 5,85 & 586280 & 5,71 & 2,5380 \\
\hline 24 serviços & 75068 & 100 & $25.471 .329,17$ & 100 & 10.261840 & 100 & 2,4821 \\
\hline
\end{tabular}


Em 2005 algumas macrorregionais gastaram e aumentaram o valor per capita, extrapolando o valor referência do Estado do Paraná que foi de $\mathrm{R} \$ 2,48$. A macrorregional Metropolitana apresentou a maior concentração populacional com 34,19\% dos habitantes do Estado do Paraná e a macrorregional Norte apresentou o maior gasto per capita com $\mathrm{R} \$ 3,40$ (Tabela 2 ).

Tabela 3. Distribuição dos serviços auditivos por macrorregião no Paraná, 2006.

\begin{tabular}{|c|c|c|c|c|c|c|c|}
\hline Macrorregional & Freq & $\%$ & Gasto & $\%$ & População & $\%$ & $\begin{array}{c}\text { Gasto per } \\
\text { capita }\end{array}$ \\
\hline $\begin{array}{l}\text { Metropolitana } \\
\text { (5 serviços) }\end{array}$ & 21859 & 35,73 & $4.912 .313,14$ & 28,35 & 3590933 & 34,6 & 1,3679 \\
\hline $\begin{array}{l}\text { Campos Gerais } \\
\text { ( } 2 \text { serviços) }\end{array}$ & 7464 & 12,2 & $1.678 .518,98$ & 9,69 & 1060283 & 10,2 & 1,583 \\
\hline $\begin{array}{l}\text { Oeste } \\
\text { (5 serviços) }\end{array}$ & 10347 & 16,92 & $3.663 .067,98$ & 21,14 & 1820242 & 17,5 & 2,0124 \\
\hline $\begin{array}{l}\text { Noroeste } \\
\text { (3 serviços) }\end{array}$ & 6123 & 10 & $3.024 .562,82$ & 17,45 & 1642684 & 15,8 & 1,8412 \\
\hline $\begin{array}{l}\text { Norte } \\
\text { (4 serviços) }\end{array}$ & 12309 & 20,12 & $3.619 .243,17$ & 20,89 & 1687066 & 16,2 & 2,1452 \\
\hline $\begin{array}{l}\text { Centro } \\
\text { (1 serviço) }\end{array}$ & 3075 & 5,03 & $429.685,36$ & 2,48 & 586200 & 5,65 & 0,733 \\
\hline 20 serviços & 61177 & 100 & $17.327 .391,45$ & 100 & 10387408 & 100 & 1,6681 \\
\hline
\end{tabular}

Em 2006 o valor per capita de referência do Estado do Paraná foi de $\mathrm{R} \$$ 1,66 observando que houve diminuição no teto financeiro pelo governo federal, porém, percebe-se que as macrorregionais que aumentaram seu gasto em 2005, também o fizeram em 2006, mesmo com redução da frequência, através dos procedimentos e gastos financeiro proposto pela Secretaria Estadual de Saúde (SESA) (Tabela 3).

\section{CONSIDERAÇÕES FINAIS}

O SUS é uma rede hierarquizada, regionalizada e descentralizada sob comando único em cada nível de governo: federal, estadual e municipal. É, pois, um sistema complexo, dinâmico e em constante evolução. Atendendo as diretrizes do SUS, os recursos federais devem ser rateados entre os Estados e Municípios, a fim de que esses entes federativos cuidem da saúde, não apenas de seus munícipes, mas, também, da população referenciada.

É fundamental a identificação dos fluxos existentes, delineando as regiões a serem reguladas, e que nem sempre correspondem às divisões administrativas 
estabelecidas pelas macrorregiões e por gestores.

Segundo o Conselho Nacional de Secretários Municipais de Saúde (CONASEMS) o gasto em saúde no Brasil é de U\$ 145 per capita/ano em valores de novembro de 2006 . Equivale a $45 \%$ do gasto nacional em saúde, 55\% correspondem aos gastos do setor privado.

Para garantir os princípios do SUS e a constante melhoria dos serviços prestados, e a qualidade de vida dos sujeitos e coletividades, entende-se que é urgente superar a cultura administrativa fragmentada e desfocada dos interesses e das necessidades da sociedade, evitando o desperdício de recurso público, reduzindo a superposição de ações e, consequentemente, aumentando a eficiência e a efetividade das políticas públicas existentes.

\section{REFERÊNCIAS}

1. World Health Organization. Deafness an hearing impairment. FactSheet, Geneva, n.300, mar. 2006. Disponível em http://who.int/medacentre/factsheets/fs300 Len/index.html acesso em 15 de setembro de 2008.

2. Bustamante MT, Alves MJM. Prevalência de perda auditiva induzida por ruído em empresa metalúrgica. Rev. Saúde Pública 2005, 39(2):238-244. http://dx.doi.org/10.1590/S0034$\underline{89102005000200015}$

3. IBGE. Estudo e Pesquisa Informações Demográficas e Socioeconômicas In:
Síntese dos Indicadores Sociais. Rio de Janeiro, 2006, 19:216-244.

4. Brasil. Ministério da Saúde. Portaria 432 SAS/MS de 14 de novembro de 2000. Disponível em http://www.dtr.saude.gov.br acesso em 12 de fevereiro de 2007.

5. Brasil. Ministério da Saúde. Portaria 2073 GM/MS de 28 de setembro de 2004. Disponível em http://www.dtr.saude.gov.br acesso em 12 de fevereiro de 2007.

6. Brasil. Ministério da Saúde. Portaria 587 SAS/MS de 07 de outubro de 2004. Disponível em http://www.dtr.saude.gov.br acesso em 11 de janeiro de 2007.

7. Goiás. Secretaria de Estado da Saúde. Superintendência de Controle e Avaliação Técnica da Saúde de Goiás. Manual de Normas e procedimentos de Auditoria. Goiânia: SES/SCATS, 2005. 89p.

8. Nonnenmacher $\mathrm{CL}$, Weiller $\mathrm{TH}$, Oliveira SG. Acesso à saúde: limites vivenciados por usuários do SUS na obtenção de um direito. Cienc. Cuid. Saúde 2011, 10(2):248-255.

9. Brasil. Ministério da Saúde. Departamento de Regulação, Avaliação e Controle de Sistemas. Departamento Nacional de Auditoria do SUS. Curso básico de regulação, controle, avaliação e auditoria do SUS. Brasília: Ministério da Saúde, 2006. $256 p$.

10. Remor. LC. Controle, Avaliação e Auditoria do Sistema Único de Saúde. [Dissertação]. Florianópolis (SC): Mestrado em Engenharia de Produção, Universidade Federal de Santa Catarina-UFSC, 2002. 202 p. 
11. Brasil. Ministério da Saúde. Departamento Nacional de Auditoria do SUS. Orientações técnicas sobre o Sistema de Informações Ambulatoriais (SIA) e Sistema de Informações Hospitalares (SIH)/ Ministério da Saúde, Departamento Nacional de Auditoria do SUS - Brasília: Ministério da Saúde, 2002.

12. Brasil. Ministério da Saúde. Nota técnica Informativa $n^{\circ} 004$. Gastos com saúde auditiva, 2003.

13. Freitas CDC, Julio M. Hearing aids fitting process in users that are seen in a federal public institution: part I-results and implications with the amplification device. Rev. Bras. Otorrinolaringol. 2007, 6:744-751. http://dx.doi.org/10.1590/S0034$\underline{72992007000600005}$

Recebido para publicação em 19/08/2014

Revisado em 28/08/2014

Aceito em 19/09/2014 